\title{
Variational calculations with improved energy functionals in gauge theories
}

\author{
C. Heinemann, ${ }^{1}$ E. Iancu, ${ }^{2}$ C. Martin, ${ }^{3}$ and D. Vautherin ${ }^{1}$ \\ ${ }^{1}$ LPNHE, LPTPE, Universités Paris VI/VII, case 127, F-75252, Paris Cedex 05, France \\ ${ }^{2}$ Theory Division, CERN, CH-1211 Geneva 23, Switzerland \\ ${ }^{3}$ Institut de Physique Nucléaire, F-91406, Orsay Cedex, France
}

(Received 24 November 1999; published 10 May 2000)

\begin{abstract}
For an $S U(N)$ Yang-Mills theory, we present variational calculations using Gaussian wave functionals combined with an approximate projection on gauge invariant states. The projection amounts to correcting the energy of the Gaussian states by subtracting the spurious energy associated with gauge rotations. Based on this improved energy functional, we perform variational calculations of the interaction energy in the presence of external electric and magnetic fields. We verify that the ultraviolet behavior of our approximation scheme is consistent, as it should be, with that expected from perturbation theory. In particular, we recover in this variational framework the standard one-loop beta function, with a transparent interpretation of the screening and anti-screening contributions.
\end{abstract}

PACS number(s): 11.15.Tk, 12.38.Lg, 14.70.Dj

\section{INTRODUCTION}

The functional Schrödinger picture has proven to be a privileged tool in exploring a rich variety of aspects of gauge theories which are beyond the scope of perturbation theory [1]. It is a useful starting point for developing nonperturbative calculations based on the variational approach. In the case of scalar field theories, static as well as dynamical variational calculations have been performed by using trial wave functionals of the Gaussian type [2-8]. In the case of gauge theories, some early investigations along these lines can be found in [9-12]. However, the application of variational methods to gauge theories is generally plagued by the difficulty to implement in a calculable way the requirement of gauge invariance of physical states [12-14].

Gaussian wave functionals allow for analytic calculations, but are not gauge invariant except in the Abelian case. In principle, one can construct gauge invariant states by averaging Gaussian wave functionals over all gauge rotations. This results in an effective non-linear sigma model where the fields are the group elements of the gauge transformations [14]. However, to make progress with this theory, further approximations are necessary both in the choice of the kernel of the Gaussian and in the evaluation of the functional integrals over the gauge group [14-18]. Such approximations, which go beyond the variational principle, are not always under control. In particular, in the perturbative regime, they fail to completely reproduce the one-loop beta function [15] (see also $[16,18]$ ).

In this paper we shall propose a different strategy which is inspired by techniques developed in 1962 by Thouless and Valatin [19] to deal with the restoration of rotational invariance when deformed solutions are obtained in nuclear Hartree-Fock calculations. Rather than using gauge invariant variational states, we shall limit ourselves to Gaussian wave functionals, but we shall correct the associated energy functional by a non-local term, which approximately corresponds to the energy gain when projecting on gauge invariant states. In the Abelian case, this amounts to removing the contribution of the longitudinal part of the Gaussian kernel to the energy. In Yang-Mills theory, the corrective energy term is itself determined by the variational principle, and the ensuing variational calculation is a priori non-perturbative.

Our main purpose here is to provide the framework for such a calculation and verify that, in the perturbative regime (i.e., when supplemented with an expansion in powers of $g$ ), it leads to results consistent with ordinary perturbation theory. As a first check, we shall show that our variational calculation reproduces the standard one-loop beta function of Yang-Mills theory, with a transparent interpretation of the various contributions in terms of screening and antiscreening phenomena. As a further test, we shall compute the vacuum energy in the presence of an external magnetic field $B$, and find that it exhibits a minimum at a nonvanishing value of $B$, in agreement with the perturbative calculation in [20].

The paper is organized as follows. In Sec. II we briefly review the functional Schrödinger picture and the variational principle in field theory. In Sec. III, we present the ThoulessValatin formalism and consider, as a simple illustration, its application to quantum electrodynamics (QED). In Sec. IV we present a variational calculation of the one-loop beta function which is based on the construction of the interaction energy between external electrostatic charges. In Sec. V we consider the energy of the QCD vacuum in the presence of a constant magnetic field. This provides an alternative computation of the beta function, and also of the gluon condensate which is found to satisfy the trace anomaly relation. A summary of our results and a discussion of further possible extensions and applications are presented in Sec. VI.

\section{VARIATIONAL CALCULATIONS FOR GAUGE FIELDS}

We consider the functional Schrödinger description of $S U(N)$ Yang-Mills theory. In the temporal gauge $A_{a}^{0}=0$, the canonical coordinates are the vector potentials $A_{a}^{i}(\mathbf{x})$ and the electric fields $E_{a}^{i}(\mathbf{x})$, which we shall often write as color matrices in the adjoint representation: e.g., $A^{i} \equiv A_{b}^{i} T^{b}$ (the color indices $a, b, \ldots$ run from 1 to $N^{2}-1$ ). The generators $T^{a}$ of the color group are taken to be Hermitian and traceless; they satisfy 


$$
\left[T^{a}, T^{b}\right]=i f^{a b c} T^{c}, \operatorname{Tr}\left(T^{a} T^{b}\right)=N \delta^{a b},\left(T^{a}\right)_{b c}=-i f^{a b c} .
$$

The Hamiltonian density reads ( $g$ denotes the coupling constant)

$$
\mathcal{H}(\mathbf{x})=\frac{1}{2}\left\{g^{2} E_{a}^{i} E_{a}^{i}(\mathbf{x})+\frac{1}{g^{2}} B_{a}^{i} B_{a}^{i}(\mathbf{x})\right\},
$$

with the color magnetic field

$$
\mathbf{B}_{a}=\boldsymbol{\nabla} \times \mathbf{A}_{a}+\frac{1}{2} f_{a b c} \mathbf{A}_{\mathbf{b}} \times \mathbf{A}_{\mathbf{c}} .
$$

Note that our conventions are such that the QCD coupling constant is absorbed in the normalization of the vector potentials. With these conventions, the covariant derivative reads $D_{i}=\partial_{i}-i A_{i}$, and the electric fields $E_{a}^{i}$ are canonically conjugate to the vector potentials $A_{a}^{i}:\left[E_{a}^{i}(\mathbf{x}), A_{b}^{j}(\mathbf{y})\right]$ $=i \delta^{i j} \delta_{a b} \delta^{(3)}(\mathbf{x}-\mathbf{y})$.

In the Schrödinger representation, the states are represented by functionals of $A_{a}^{i}(\mathbf{x}), \Psi[\mathbf{A}]$, and the electric field is acting on such states by functional differentiation:

$$
E_{a}^{i}(\mathbf{x}) \Psi[\mathbf{A}]=i \frac{\delta}{\delta A_{a}^{i}(\mathbf{x})} \Psi[\mathbf{A}] .
$$

The Hamiltonian $H$ commutes with the generator $\mathcal{G}$ of timeindependent gauge transformations,

$$
\mathcal{G}(\mathbf{x}) \equiv \boldsymbol{\nabla} \cdot \mathbf{E}(\mathbf{x})+i\left[A^{i}, E^{i}\right],
$$

so it is possible to diagonalize $H$ and $\mathcal{G}$ simultaneously.

The physical states are constrained by Gauss' law:

$$
\mathcal{G}(\mathbf{x}) \Psi[\mathbf{A}]=0,
$$

which is the requirement of gauge invariance. [More precisely, Eq. (2.5) shows that physical states must be invariant under "small" (i.e., topologically trivial [1]) gauge transformations. We shall not be concerned with the topological aspects of the gauge symmetry in what follows.] More generally, in the presence of matter fields represented by an external color source with density $\rho^{a}$, Gauss' law gets modified as follows:

$$
\mathcal{G}_{a}(\mathbf{x}) \Psi[\mathbf{A}]=\rho_{a}(\mathbf{x}) \Psi[\mathbf{A}]
$$

The ground state of QCD is the eigenstate $\Psi_{v a c}$ of $H$ of minimal energy which satisfies Gauss' constraint (2.5). It can be constructed, at least in principle, by using the Ritz variational principle, which states that

$$
\langle H\rangle \equiv \frac{\langle\Psi|H| \Psi\rangle}{\langle\Psi \mid \Psi\rangle} \geqslant E_{v a c},
$$

with the minimum achieved for $\Psi=\Psi_{v a c}$. Here, $\Psi[\mathbf{A}]$ is any wave functional from the physical Hilbert space [i.e., which satisfies Eq. (2.5)], and $E_{v a c}$ is the energy of the ground state $\Psi_{v a c}$, assumed to be non-degenerate. In practice, however, one has to restrict oneself to Gaussian wave functionals, the only ones which allow an analytical computation of $\langle H\rangle$. These have the form

$$
\begin{aligned}
\Psi_{0}[\mathbf{A}]= & \mathcal{N}^{-1} \exp \left\{-\frac{1}{4 g^{2}} \int \mathrm{d}^{3} x \mathrm{~d}^{3} y\left[A_{a}^{i}(\mathbf{x})-\bar{A}_{a}^{i}(\mathbf{x})\right]\right. \\
& \left.\times\left(G^{-1}\right)_{i j}^{a b}(\mathbf{x}, \mathbf{y})\left[A_{b}^{j}(\mathbf{y})-\bar{A}_{b}^{j}(\mathbf{y})\right]\right\},
\end{aligned}
$$

where the background field $\bar{A}_{a}^{i}(\mathbf{x})$ and the kernel $G^{-1}$ [with matrix elements $\left.\left(G^{-1}\right)_{i j}^{a b}(\mathbf{x}, \mathbf{y})\right]$ are the variational parameters. We expect that $\bar{A}=0$ in the vacuum state, and this is the case that we shall consider mostly in this paper. Still, non-vanishing values of $\bar{A}$ will be also considered, in Sec. V below, in a study of the vacuum stability in the presence of a background color magnetic field (in the same spirit as, e.g., in Ref. [20]).

The expectation value of the Hamiltonian density in the Gaussian state $\Psi_{0}$ is [12]

$$
\begin{aligned}
\left\langle\Psi_{0}|\mathcal{H}(\mathbf{x})| \Psi_{0}\right\rangle= & \frac{1}{2 g^{2}} \overline{\mathbf{B}} \cdot \overline{\mathbf{B}}(\mathbf{x})+\frac{1}{8} \operatorname{Tr}\left\langle\mathbf{x}\left|G^{-1}\right| \mathbf{x}\right\rangle \\
& +\frac{1}{2} \operatorname{Tr}\langle\mathbf{x}|K G| \mathbf{x}\rangle+\frac{g^{2}}{8}\left(\operatorname{Tr}\left\{S_{i} T^{a}\langle\mathbf{x}|G| \mathbf{x}\rangle\right\}\right)^{2} \\
& +\frac{g^{2}}{4} \operatorname{Tr}\left\{S^{i} T^{a}\langle\mathbf{x}|G| \mathbf{x}\rangle S^{i} T^{a}\langle\mathbf{x}|G| \mathbf{x}\rangle\right\}
\end{aligned}
$$

In this equation $\overline{\mathbf{B}}$ is the magnetic field associated with the center $\overline{\mathbf{A}}$ and $S^{i}$ is the spin 1 matrix whose elements $(j, k)$ are given by $i \varepsilon_{i j k}$. The notation $\operatorname{Tr}$ in Eq. (2.9) implies a summation over the discrete (color and spatial) indices. For instance, $\operatorname{Tr}\left\langle\mathbf{x}\left|G^{-1}\right| \mathbf{x}\right\rangle=\Sigma_{i, a}\left(G^{-1}\right)_{i i}^{a a}(\mathbf{x}, \mathbf{x})$. Finally, the operator $K$ is the second derivative of the classical energy with respect to the center $\bar{A}_{i}^{a}$. It reads, in matrix notation,

$$
K=(-i \mathbf{S} \cdot \mathcal{D})^{2}-\mathbf{S} \cdot \overline{\mathbf{B}},
$$

where

$$
\mathcal{D}^{i} \equiv \partial^{i}-i \bar{A}^{i}
$$

denotes the covariant derivative defined by the background field $\bar{A}^{i} \equiv \bar{A}_{a}^{i} T^{a}$. In particular,

$$
K_{i j}(p)=p^{2} \delta_{i j}-p_{i} p_{j} \quad \text { for } \quad \bar{A}=0 .
$$

In the case of non-Abelian gauge theories, however, Gaussian functionals such as Eq. (2.8) suffer from a major drawback: they do not satisfy the requirement of gauge invariance (2.5). It is in principle possible to construct gauge invariant states by projection, i.e. by averaging a Gaussian functional over all its gauge transformations. This is achieved by means of the formula 


$$
\Psi[\mathbf{A}]=\frac{1}{\mathcal{N}} \int \mathcal{D}[U(\mathbf{x})] \Psi_{U}[\mathbf{A}],
$$

where the functional integration is performed over the unitary $N \times N$ matrix field $U(\mathbf{x})$, with the adequate group invariant measure, and $\mathcal{N}$ is a normalization factor. The integrand in Eq. (2.13) is the gauge transform of the Gaussian state $\Psi_{0}[\mathbf{A}]$ :

$$
\Psi_{U}[\mathbf{A}]=\Psi_{0}\left[U \mathbf{A} U^{+}+i U \boldsymbol{\nabla} U^{+}\right]
$$

The expectation value $E_{P}$ of the energy in the projected state is given by the following formula:

$$
E_{P}=\frac{\int \mathcal{D}[U(\mathbf{x})]\left\langle\Psi_{0}|H| \Psi_{U}\right\rangle}{\int \mathcal{D}[U(\mathbf{x})]\left\langle\Psi_{0} \mid \Psi_{U}\right\rangle},
$$

which should replace Eq. (2.9) in practical calculations. Unfortunately, Eq. (2.15) cannot be evaluated in closed form because the functional integral over the group is not Gaussian. Various approximations to Eq. (2.15) have been considered in Refs. [14-18]. In what follows, we shall propose a different approximation method which is inspired from techniques used in nuclear physics to calculate the zero point rotational energy of deformed nuclei [19,21-23].

\section{APPROXIMATE PROJECTION}

The fact that our variational ground state, namely the Gaussian $\Psi_{0}$ in Eq. (2.8), is not gauge invariant introduces a spurious degeneracy in the problem: $\Psi_{0}$ is degenerate with all its gauge transforms defined in Eq. (2.14). This leads to the existence of spurious excitations of zero energy which correspond to rotations of $\Psi_{0}$ in the gauge space; these are, of course, the Goldstone bosons associated with the spontaneous breaking of the gauge symmetry by $\Psi_{0}$. Accordingly, the expectation value (2.9) of the Hamiltonian in the $d e$ formed state $\Psi_{0}$ includes unphysical contributions expressing the kinetic energy of the gauge rotations. The ThoulessValatin formalism [19] provides us with a method to estimate, and thus subtract away, such unphysical contributions.

\section{A. Case of rotations}

This formalism is best explained by the example of the collective rotations of a deformed nucleus. There, the equivalent of our present variational calculation with Gaussian wave functionals is the so-called Hartree-Fock approximation where the nuclear wave function is represented by a Slater determinant formed with $A$ single-particle wave functions $\varphi_{k}(x)$ (for $A$ nucleons) [23]. The latter are determined by solving the Hartree-Fock equations, i.e., the variational equations obtained by minimizing the expectation value of the Hamiltonian in the subspace of Slater determinants. Although the Hamiltonian $H$ is rotationally invariant, the Hartree-Fock equations may lead to non-invariant solutions describing nuclear deformations. If this is the case, then the spectrum develops a ground state rotational band,

$$
E_{J}=E_{0}+\frac{J(J+1)}{2 I},
$$

where $J(J+1)$ is the eigenvalue of the angular momentum operator $\mathbf{J}^{2}$ and $I$ is the moment of inertia. One may then conclude that the operator

$$
\widetilde{H} \equiv H-\frac{\mathbf{J}^{2}}{2 I}
$$

is the Hamiltonian of the intrinsic (that is, non-rotational) motion: to the ground state rotational band of $H$ corresponds now a single eigenvalue $E_{0}$ of $\widetilde{H}$. Then, Eq. (3.2) provides an approximate separation of the dynamics into intrinsic and rotational motion, which is reminiscent of the familiar separation of the center-of-mass motion by the formula

$$
\widetilde{H} \equiv H-\frac{\mathbf{P}^{2}}{2 M},
$$

where

$$
\mathbf{P}=\mathbf{p}_{1}+\mathbf{p}_{2}+\ldots+\mathbf{p}_{A}
$$

is the total momentum operator, and $M=A m$ is the nuclear mass. Thus, a mean field description of the intrinsic motion can be given by performing variational (or Hartree-Fock) calculations for the substracted Hamiltonian $\widetilde{H}$. The only question is, what is the value of the moment-of-inertia parameter $I$ ?

One can answer this question by studying rotations of the deformed mass distribution. Assume that the nucleus has axial symmetry with respect to the $z$ axis, and consider a uniform rotation with angular velocity $\omega$ about the $x$ axis. If $\Psi_{\omega}(t)$ is the exact (time-dependent) state describing such a rotation, then

$$
\Psi_{\omega}(t)=\mathrm{e}^{-i \omega t J_{x}} \mathrm{e}^{-i E_{\omega} t} \Psi_{\omega}(0),
$$

which satisfies the time-dependent Schrödinger equation

$$
i \frac{\partial \Psi_{\omega}}{\partial t}=H \Psi_{\omega},
$$

provided $\Psi_{\omega}(0)$ is a solution to the following timeindependent problem:

$$
\left(H-\omega J_{x}\right) \Psi_{\omega}(0)=E_{\omega} \Psi_{\omega}(0) .
$$

This is, of course, just the familiar transformation to the rotating frame of reference, which leads us to consider the variational problem for the following Hamiltonian:

$$
H_{\omega}=H-\omega J_{x} .
$$

This is equivalent to a constrained variational problem with the subsidiary condition that $\left\langle J_{x}\right\rangle$ has a given value; $\omega$ plays then the role of the Lagrange multiplier. The constrained 
Hartree-Fock calculation determines the optimal independent nucleon wave function $\Psi_{\omega} \equiv \Psi_{\omega}(0)$ in the rotating frame. (As $\omega \rightarrow 0, \Psi_{\omega} \rightarrow \Psi_{0}$ which is the deformed Hartree-Fock ground state.) Once the optimal wave function is found it is possible to obtain an estimate of the moment of inertia $I$ of the nucleus by considering the limit

$$
I=\lim _{\omega \rightarrow 0} \frac{\left\langle\Psi_{\omega}\left|J_{x}\right| \Psi_{\omega}\right\rangle}{\omega} .
$$

This finally allows us to estimate the zero-point rotational energy $\Delta E_{T V}$ in the deformed state $\Psi_{0}$ :

$$
\Delta E_{T V}=\frac{\left\langle\Psi_{0}\left|\mathbf{J}^{2}\right| \Psi_{0}\right\rangle}{2 I} .
$$

Equation (3.10) is the expected gain in energy when projecting the deformed Hartree-Fock ground state $\Psi_{0}$ onto a rotationally invariant state. That is, the corrected average energy after projection, which is the energy of the intrinsic motion [cf. Eq. (3.2)], reads

$$
\widetilde{E}=E_{0}-\Delta E_{T V}
$$

where $E_{0} \equiv\left\langle\Psi_{0}|H| \Psi_{0}\right\rangle$.

The Thouless-Valatin method is an approximation which is expected to be valid for large deformations or, more precisely, when the deformation produces a large expectation value of the square of the angular momentum in the HartreeFock ground state [21]. Indeed in this case it can be shown that the projection onto invariant states can be accurately performed because the overlap between two states differing in their orientation by an angle $\theta$ is sharply peaked near $\theta$ $=0$, thus allowing for an expansion in the vicinity of this point [22].

\section{B. Application to QED}

When going to gauge theories in the variational method, the Hartree-Fock ground state $\Psi_{0}$ is replaced by the Gaussian trial wave functional [cf. Eq. (2.8)], and the angular momentum operator is replaced by the generator of the gauge transformations, $\mathcal{G}^{a}(\mathbf{x})$ [cf. Eq. (2.4)].

As a first illustration let us consider a variational calculation in QED, with the Gaussian variational ansatz

$$
\Psi_{0}[\mathbf{A}]=\mathcal{N}^{-1} \exp \left\{-\left\langle A\left|\frac{1}{4 G}\right| A\right\rangle\right\},
$$

where $\mathcal{N}=(\operatorname{det} G)^{1 / 4}$ and the expression in the exponent is a condensed notation for the convolution

$$
\left\langle A\left|\frac{1}{4 G}\right| A\right\rangle \equiv \frac{1}{4} \int \mathrm{d}^{3} x \mathrm{~d}^{3} y A^{i}(\mathbf{x}) G_{i j}^{-1}(\mathbf{x}, \mathbf{y}) A^{j}(\mathbf{y}) .
$$

This wave functional is gauge invariant provided its kernel $G^{-1}$ is transverse: $\partial_{i} G_{i j}^{-1}=0$. Let us assume, however, that this is not the case, and see what the Thouless-Valatin cor- rection would predict in this case. The operator playing the role of the angular momentum is the charge density operator

$$
\mathcal{G}(\mathbf{x})=\nabla \cdot \mathbf{E}(\mathbf{x})
$$

and the generalization of the Thouless-Valatin formula (3.10) for the energy correction reads

$$
\Delta E_{T V}=\int \mathrm{d}^{3} x \mathrm{~d}^{3} y\left\langle\Psi_{0}|\mathcal{G}(\mathbf{x}) \mathcal{G}(\mathbf{y})| \Psi_{0}\right\rangle\left\langle\mathbf{x}\left|\frac{1}{2 \mathcal{I}}\right| \mathbf{y}\right\rangle,
$$

where the "moment of inertia" $\mathcal{I}$ is now a matrix in coordinate space, with matrix elements $\langle\mathbf{x}|\mathcal{I}| \mathbf{y}\rangle \equiv \mathcal{I}(\mathbf{x}, \mathbf{y})$. This is obtained via a constrained variational calculation with Hamiltonian $H_{\omega}=H-H_{e x t}$, where

$$
H=\frac{1}{2} \int \mathrm{d}^{3} x\left\{\mathbf{E}^{2}(\mathbf{x})+(\boldsymbol{\nabla} \times \mathbf{A})^{2}\right\}
$$

and the external constraint

$$
H_{e x t}=\int \mathrm{d}^{3} x \omega(\mathbf{x}) \boldsymbol{\nabla} \cdot \mathbf{E}(\mathbf{x}) .
$$

In the present context, the Lagrange multiplier $\omega(\mathbf{x})$ plays the role of the temporal component $A^{0}(\mathbf{x})$ of the gauge vector potential. The solution to this constrained variational problem is of the form ${ }^{1}$

$$
\Psi_{\omega}[\mathbf{A}]=\exp \{-i\langle\mathbf{F} \mid \mathbf{A}\rangle\} \Psi_{0}[\mathbf{A}]
$$

where the vector field $\mathbf{F}(\mathbf{x})$ is a new variational parameter, which expresses the expectation value of the electric field in the state (3.18), $F^{i}=\left\langle\Psi_{\omega}\left|E^{i}\right| \Psi_{\omega}\right\rangle$, and is determined by minimizing

$$
\begin{aligned}
E_{\omega} & \equiv\left\langle\Psi_{\omega}\left|H-H_{\text {ext }}\right| \Psi_{\omega}\right\rangle \\
& =E_{0}+\frac{1}{2} \int \mathrm{d}^{3} x(\mathbf{F}+\nabla \omega)^{2}-\frac{1}{2} \int \mathrm{d}^{3} x(\nabla \omega)^{2} .
\end{aligned}
$$

We have denoted here ( $V$ is the total volume of the space)

$$
\begin{aligned}
E_{0} & \equiv\left\langle\Psi_{0}|H| \Psi_{0}\right\rangle \\
& =\frac{V}{2} \int \frac{\mathrm{d}^{3} p}{(2 \pi)^{3}}\left\{\frac{1}{4} G_{i i}^{-1}(\mathbf{p})+\left(p^{2} \delta_{i j}-p_{i} p_{j}\right) G_{i j}(\mathbf{p})\right\} .
\end{aligned}
$$

\footnotetext{
${ }^{1}$ Equation (3.18) can be simply understood by recalling that, in the presence of a constraint of the form $H_{e x t}=\alpha p$, the ground state wave function of an harmonic oscillator is modified by a factor $\exp \left(i p_{0} x\right)$, but its width remains unchanged.
} 
Note that the magnetic piece of the energy (3.20) (the second term between parentheses) involves only the transverse components of $G_{i j}$, while the electric piece involves also its longitudinal component.

The functional $E_{\omega}[\mathbf{F}]$ in Eq. (3.19) attains its minimum for $\mathbf{F}=-\nabla \omega$, in which case

$$
\left\langle\Psi_{\omega}|\boldsymbol{\nabla} \cdot \mathbf{E}| \Psi_{\omega}\right\rangle=\boldsymbol{\nabla} \cdot \mathbf{F}=-\Delta \omega .
$$

According to Eq. (3.9), the moment of inertia is obtained as (with $\langle\boldsymbol{\nabla} \cdot \mathbf{E}\rangle_{\omega} \equiv\left\langle\Psi_{\omega}|\boldsymbol{\nabla} \cdot \mathbf{E}| \Psi_{\omega}\right\rangle$ ):

$$
\begin{aligned}
\langle\mathbf{x}|\mathcal{I}| \mathbf{y}\rangle & =\frac{\delta\langle\boldsymbol{\nabla} \cdot \mathbf{E}(\mathbf{x})\rangle_{\omega}}{\delta \omega(\mathbf{y})} \\
& =\langle\mathbf{x}|-\Delta| \mathbf{y}\rangle,
\end{aligned}
$$

whose inverse is simply the Coulomb propagator

$$
\left\langle\mathbf{x}\left|\frac{1}{I}\right| \mathbf{y}\right\rangle=\frac{1}{4 \pi|\mathbf{x}-\mathbf{y}|} .
$$

We thus obtain the following expression for the ThoulessValatin correction (3.15) in QED:

$$
\Delta E_{T V}=\frac{1}{2} \int \mathrm{d}^{3} x \mathrm{~d}^{3} y\left\langle\Psi_{0}|\boldsymbol{\nabla} \cdot \mathbf{E}(\mathbf{x}) \boldsymbol{\nabla} \cdot \mathbf{E}(\mathbf{y})| \Psi_{0}\right\rangle \frac{1}{4 \pi|\mathbf{x}-\mathbf{y}|},
$$

which is recognized as the electrostatic energy in the state $\Psi_{0}$. For the Gaussian state (3.12), this gives

$$
\Delta E_{T V}=\frac{V}{8} \int \frac{\mathrm{d}^{3} p}{(2 \pi)^{3}} \frac{p_{i} p_{j}}{p^{2}} G_{i j}^{-1}(\mathbf{p}),
$$

which simply subtracts the longitudinal piece of the electric energy in Eq. (3.20).

The corrected energy $\widetilde{E} \equiv E_{0}-\Delta E_{T V}$ reads therefore [cf. Eqs. (3.20) and (3.25)]

$$
\widetilde{E}=V \int \frac{\mathrm{d}^{3} p}{(2 \pi)^{3}}\left\{\frac{1}{4} G_{T}^{-1}(p)+p^{2} G_{T}(p)\right\},
$$

and involves only the transverse piece $G_{T} \equiv \frac{1}{2}\left(\delta_{i j}\right.$ $-\hat{p}_{i} \hat{p}_{j}$ ) $G_{i j}$ of the kernel $G_{i j}$ (we have written here $p \equiv|\mathbf{p}|$ and $\left.\hat{p}_{i} \equiv p_{i} / p\right)$. Then, the variational equation $\delta \widetilde{E} / \delta G_{T}=0$ gives $G_{T}$ in the expected form

$$
G_{T}(p)=\frac{1}{2 p} .
$$

Together, Eqs. (3.26) and (3.27) yield an energy density

$$
\widetilde{E}=V \int \frac{\mathrm{d}^{3} p}{(2 \pi)^{3}}|\mathbf{p}|,
$$

which is indeed the correct result for the QED ground state [1]. Thus, in the case of QED, the approximate projection method of Thouless and Valatin correctly subtracts the con- tribution of the unphysical, gauge, degrees of freedom from the average energy. Actually, since QED without fermions is a free theory, the variational solution above coincides with the exact solution [1]: the exact ground state is a Gaussian wave functional such as Eq. (3.12) with a transverse kernel determined by Eq. (3.27):

$$
\begin{aligned}
\Psi_{v a c}[\mathbf{A}] & =\exp \left\{-\int \frac{\mathrm{d}^{3} p}{(2 \pi)^{3}} \frac{p}{2} A^{i}(\mathbf{p})\left(\delta_{i j}-\hat{p}_{i} \hat{p}_{j}\right) A^{i}(-\mathbf{p})\right\} \\
& =\exp \left\{-\int \mathrm{d}^{3} x \mathrm{~d}^{3} y \frac{\mathbf{B}(\mathbf{x}) \cdot \mathbf{B}(\mathbf{y})}{4 \pi^{2}|\mathbf{x}-\mathbf{y}|^{2}}\right\} .
\end{aligned}
$$

Moreover, wave functionals of the type shown in Eq. (3.18) - i.e., Gaussian states with a transverse kernel and a non-trivial phase factor-correspond to physical charged states, i.e., states of the quantum Maxwell theory in the presence of static, classical, external sources. Indeed, any such a state (which we denote here as $\Psi_{c}$ ) satisfies

$$
\boldsymbol{\nabla} \cdot \mathbf{E}(\mathbf{x}) \Psi_{c}[\mathbf{A}]=\rho(\mathbf{x}) \Psi_{c}[\mathbf{A}],
$$

with the charge density $\rho(\mathbf{x})=\boldsymbol{\nabla} \cdot \mathbf{F}(\mathbf{x})$. The corresponding energy includes the Coulomb energy, as expected:

$$
\begin{aligned}
\left\langle\Psi_{c}|H| \Psi_{c}\right\rangle & =E_{0}+\frac{1}{2} \int \mathrm{d}^{3} x \mathbf{F}^{2}(\mathbf{x}) \\
& =E_{0}+\frac{1}{2} \int \mathrm{d}^{3} x \mathrm{~d}^{3} y \frac{\rho(\mathbf{x}) \rho(\mathbf{y})}{4 \pi|\mathbf{x}-\mathbf{y}|}
\end{aligned}
$$

Non-Abelian charged states will be considered in Sec. IV B below.

For other applications and a more complete study of the Thouless-Valatin method in the context of quantum field theory, see [24].

\section{Approximate projection in QCD}

Let us now consider the case of non-Abelian gauge theories. The corresponding "moment of inertia" is now a color matrix defined as the polarization tensor [cf. Eq. (2.4)]

$$
\mathcal{I}^{a b}(\mathbf{x}, \mathbf{y})=\left.\frac{\delta\left\langle\mathcal{G}^{a}(\mathbf{x})\right\rangle_{\omega}}{\delta \omega^{b}(\mathbf{y})}\right|_{\omega=0},
$$

in the presence of an external constraint

$$
\begin{aligned}
H_{\omega} & =H-H_{\text {ext }} \\
H_{\text {ext }} & =\int \mathrm{d}^{3} x \omega^{a}(\mathbf{x}) \mathcal{G}^{a}(\mathbf{x}) .
\end{aligned}
$$

The analogue of Eq. (3.10), i.e. the gain in energy when projecting a wave functional $\Psi_{0}[\mathbf{A}]$ onto the subspace of gauge invariant states, reads 


$$
\Delta E_{T V}=\int \mathrm{d}^{3} x \mathrm{~d}^{3} y\left\langle\Psi_{0}\left|\mathcal{G}^{a}(\mathbf{x}) \mathcal{G}^{b}(\mathbf{y})\right| \Psi_{0}\right\rangle\left\langle a, \mathbf{x}\left|\frac{1}{2 \mathcal{I}}\right| b, \mathbf{y}\right\rangle
$$

The energy functional to be used in variational calculations is therefore

$$
\widetilde{E}=E_{0}-\Delta E_{T V} .
$$

It is also possible to perform a projection on a subspace with a given color charge distribution $\left\langle\mathcal{G}^{a}(\mathbf{x})\right\rangle_{c} \neq 0$ (the subscript $c$ refers to expectation values over charged states). In such a case, however, the gauge generator appearing in the previous correction formula has to be replaced by its deviation $\hat{\mathcal{G}}$ away from the desired value:

$$
\hat{\mathcal{G}}^{a}(\mathbf{x})=\mathcal{G}^{a}(\mathbf{x})-\left\langle\mathcal{G}^{a}(\mathbf{x})\right\rangle_{c} .
$$

This modification guarantees that there is no correction for a state which is an exact eigenstate of the charge operator. The functional to be minimized in the subspace of Gaussian functionals is thus

$$
\widetilde{E}=E_{c}-E_{\mathrm{ext}}-\Delta \hat{E}_{T V},
$$

where $E_{c}=\langle H\rangle_{c}, E_{\mathrm{ext}}=\left\langle H_{\mathrm{ext}}\right\rangle_{c}$ is the energy of the external constraint generating the charged state, ${ }^{2}$ and

$$
\Delta \hat{E}_{T V}=\int \mathrm{d}^{3} x \mathrm{~d}^{3} y\left\langle\hat{\mathcal{G}}^{a}(\mathbf{x}) \hat{\mathcal{G}}^{b}(\mathbf{y})\right\rangle_{c}\left\langle a, \mathbf{x}\left|\frac{1}{2 \mathcal{I}}\right| b, \mathbf{y}\right\rangle .
$$

This procedure is again reminiscent of the elimination of the center-of-mass motion in the mean field description of a composite system of $A$ particles [19]. For a system characterized by a set of single-particle wave functions $\varphi_{1}, \varphi_{2}, \ldots, \varphi_{A}$ the optimal state in the center-of-mass frame is obtained by minimizing the functional

$$
\widetilde{E}=\langle H\rangle-\frac{\left\langle\mathbf{P}^{2}\right\rangle}{2 M} .
$$

The Thouless-Valatin prescription for the total mass $M$ in Eq. (3.39) is to use the relation $\langle\mathbf{P}\rangle \equiv M \mathbf{v}$, where $\langle\mathbf{P}\rangle$ is the expectation value of the total momentum (3.4) in the presence of the external constraint $H_{\text {ext }}=\mathbf{v} \cdot \mathbf{P}$. This prescription gives the desired result $M=m A$ where $m$ is the mass of the individual constituents. In a moving frame with velocity $\mathbf{v}$ the single particle wave functions become

$$
\varphi_{i}(\mathbf{x}) \rightarrow e^{i \chi(\mathbf{x})} \varphi_{i}(\mathbf{x}),
$$

with $\chi(\mathbf{x})=m \mathbf{v} \cdot \mathbf{x}$. Individual momentum operators in the moving frame are obtained by the gauge transformation

$$
\nabla \rightarrow \nabla+i(\nabla \chi),
$$

\footnotetext{
${ }^{2}$ That is, an exact charged state $\Psi_{c}$ is defined as an eigenstate of $H-H_{\text {ext }}$.
}

i.e. $\mathbf{p}_{i} \rightarrow \mathbf{p}_{i}-\left\langle\mathbf{p}_{i}\right\rangle$. The functional providing the adequate state at the minimum is

$$
\widetilde{E}=\langle H\rangle-\mathbf{v} \cdot \mathbf{P}-\frac{\left\langle(\mathbf{P}-\langle\mathbf{P}\rangle)^{2}\right\rangle}{2 M},
$$

in agreement with Eq. (3.37). This procedure to implement Gauss's law will be important in Sec. IV when applied to the calculation of the interaction energy of color charges.

Still, in the case of charged states, the chromostatic energy $E_{\text {chromo }}$ is given, in our approximation scheme, by the classical chromostatic energy corrected by the ThoulessValatin term:

$$
\begin{aligned}
E_{\text {chromo }}= & \int \mathrm{d}^{3} x \mathrm{~d}^{3} y\left\langle\mathcal{G}^{a}(\mathbf{x})\right\rangle_{c}\left\langle\mathcal{G}^{b}(\mathbf{y})\right\rangle_{c}\left\langle a, \mathbf{x}\left|\frac{1}{2 \mathcal{I}}\right| b, \mathbf{y}\right\rangle \\
& +\Delta \hat{E}_{T V}
\end{aligned}
$$

so that

$$
E_{\text {chromo }}=\int \mathrm{d}^{3} x \mathrm{~d}^{3} y\left\langle\mathcal{G}^{a}(\mathbf{x}) \mathcal{G}^{b}(\mathbf{y})\right\rangle_{c}\left\langle a, \mathbf{x}\left|\frac{1}{2 \mathcal{I}}\right| b, \mathbf{y}\right\rangle .
$$

(This is the analogue of using $E_{r o t}=\left\langle\mathbf{J}^{2}\right\rangle / 2 I$ as an approximation for the rotational energy of a deformed nucleus.) In the case of QED this identification is obvious in equations such as Eqs. (3.24) or (3.31).

To conclude, the central result of this section is the nonlocal energy functional (3.35) [or Eq. (3.37) in the case of charged states] which approximately corrects for the lack of gauge symmetry when working with Gaussian states. This energy functional is the starting point of the variational method we propose for gauge theories. Note that the corrective energy term $\Delta E_{T V}$ in Eq. (3.34) is a priori of a nonperturbative nature. Our aim in what follows is to check the ultraviolet behavior of this approximation scheme. We shall thus consider the variational calculations in the perturbative regime $g \ll 1$.

\section{ONE-LOOP BETA FUNCTION FROM VARIATIONAL CALCULATIONS}

In this section, we shall use Eq. (3.43) to estimate the electrostatic energy $E_{\text {chromo }}$ of a non-Abelian charged state, up to order $g^{2}$ in perturbation theory. This will allow us to recover the standard expression for the QCD beta function in the one-loop approximation.

\section{A. Moment of inertia for color rotations}

The first step is the calculation of the moment of inertia for color rotations, $\mathcal{I}_{a b}(\mathbf{x}, \mathbf{y})$, which enters Eq. (3.43). Unlike QED, where this quantity has been computed exactly [cf. Eq. (3.23)], in QCD we shall give only a perturbative estimate of $I$, valid to the order of interest (i.e., up to order $g^{2}$ ). To this aim, it is sufficient to perform variational calculations in the vicinity of the perturbative vacuum. 
To zeroth order, the vacuum of Yang-Mills theory is the same as for Maxwell theory, namely [cf. Eq. (3.29)]:

$$
\Psi_{0}[\mathbf{A}]=\mathcal{N}^{-1} \exp \left\{-\left\langle A\left|\frac{G^{-1}}{4 g^{2}}\right| A\right\rangle\right\},
$$

where

$$
\left(G^{-1}\right)_{i j}^{a b}(\mathbf{k})=\delta_{a b}\left(\delta_{i j}-\frac{k_{i} k_{j}}{k^{2}}\right) G_{\mathbf{k}}^{-1}
$$

and $G_{\mathbf{k}}^{-1}=2 k$. In the calculation of $I$ below, we shall never need to go beyond this leading order approximation for $G_{\mathbf{k}}$.

Note that, even with such a transverse kernel, the functional (4.1) is still not invariant under non-Abelian gauge tranformations; that is, this is a deformed state, according to the terminology in Sec. III. In order to compute its moment of inertia under color rotations, one has to study the response of this state to an external constraint of the form (3.33). The trial wave functional in the presence of this constraint reads

$$
\Psi_{\omega}[\mathbf{A}]=\mathcal{N}^{-1} \mathrm{e}^{-i\langle\mathbf{F} \mid \mathbf{A}\rangle} \exp \left\{-\left\langle A\left|\frac{G^{-1}}{4 g^{2}}-i \Sigma\right| A\right\rangle\right\},
$$

which involves two additional variational parameters: the vector field $F_{a}^{i}(\mathbf{x})$ (which fixes the expectation value of the electric field: $\left.\left\langle\mathbf{E}_{a}\right\rangle=\mathbf{F}_{a}\right)$ and the matrix $\Sigma_{a b}^{i j}(\mathbf{x}, \mathbf{y})$, which is taken to be transverse in its spatial indices. The emergence of $\Sigma$ is a hallmark of non-Abelian behavior [recall that $\Sigma=0$ in the corresponding Abelian problem; cf. Eq. (3.18)].

We shall shortly see that, as $\omega \rightarrow 0, F$ and $\Sigma$ are linear in $\omega$, while the first correction to $G^{-1}$ is only quadratic, ${ }^{3}$ and therefore does not matter for the calculation of $\mathcal{I}$ [cf. Eq. (3.32)]. Thus, for the present purposes, we can take $G^{-1}$ as in Eq. (4.2). The variational parameters in Eq. (4.3) are obtained by minimizing the following functional:

$$
\begin{aligned}
E_{\omega} & \equiv\left\langle\Psi_{\omega}\left|H-H_{\text {ext }}\right| \Psi_{\omega}\right\rangle \\
& =\langle H\rangle_{\omega}-\int \mathrm{d}^{3} x \quad \omega^{a}\left\langle\mathcal{G}^{a}\right\rangle_{\omega},
\end{aligned}
$$

with respect to variations in $F_{a}^{i}$ and $\Sigma_{a b}^{i j}$. A straightforward calculation yields

$$
\langle H\rangle_{\omega}=\frac{g^{2}}{2} \int \mathrm{d}^{3} x\left\{\mathbf{F}_{a} \cdot \mathbf{F}_{a}(\mathbf{x})+4 g^{2} \operatorname{Tr}\langle\mathbf{x}|\Sigma G \Sigma| \mathbf{x}\rangle\right\},
$$

where we have kept only the terms involving the variational parameters. Similarly,

$$
\left\langle\mathcal{G}^{a}(\mathbf{x})\right\rangle_{\omega}=\boldsymbol{\nabla} \cdot \mathbf{F}^{a}(\mathbf{x})-i g^{2} \operatorname{Tr}\left\langle\mathbf{x}\left|T^{a}[\Sigma, G]\right| \mathbf{x}\right\rangle .
$$

\footnotetext{
${ }^{3}$ Such quadratic corrections occur since, in contrast to QED, the non-Abelian constraint (3.33) generates a coupling between $G$ and $F$ in the variational equations.
}

After inserting these expressions into Eq. (4.4), and taking variations with respect to $F$ and $\Sigma$, we derive the following expressions for the variational parameters (in momentum space):

$$
F_{a}^{i}(\mathbf{q})=-\frac{i q^{i}}{g^{2}} \omega_{a}(\mathbf{q})
$$

and, for the transverse ${ }^{4}$ components of $\Sigma$,

$$
\begin{aligned}
\left\langle k^{\prime} b|\Sigma| k c\right\rangle= & \frac{(2 \pi)^{3}}{V} \delta\left(\mathbf{k}^{\prime}-\mathbf{k}-\mathbf{q}\right) \\
& \times \frac{1}{2 g^{2}} \omega^{a}(\mathbf{q}) f_{a b c}\left(\frac{G_{\mathbf{k}}-G_{\mathbf{k}^{\prime}}}{G_{\mathbf{k}}+G_{\mathbf{k}^{\prime}}}\right),
\end{aligned}
$$

where $V$ is the total volume and $G_{\mathbf{k}}$ is defined in Eq. (4.2).

By using Eqs. (4.6), (4.7) and (4.8), we can finally express the average color charge $\langle\mathcal{G}\rangle_{\omega}$ in terms of $\omega^{a}$. This is conveniently decomposed into an "Abelian" and a "nonAbelian" piece, as corresponding to the two pieces on the right hand side (RHS) of Eq. (4.6): $\left\langle\mathcal{G}^{a}\right\rangle_{\omega}=\rho_{A}^{a}+\rho_{N A}^{a}$, with

$$
\rho_{A}^{a}(\mathbf{q})=i \mathbf{q} \cdot \mathbf{F}^{a}(\mathbf{q})=\frac{1}{g^{2}} \mathbf{q}^{2} \omega^{a}(\mathbf{q})
$$

and, respectively,

$$
\begin{aligned}
\rho_{N A}^{a}(\mathbf{q})= & g^{2} f_{a b c} \int \frac{\mathrm{d}^{3} k}{(2 \pi)^{3}}\left(\delta_{i j}-\frac{k_{i}^{\prime} k_{j}^{\prime}}{k^{\prime 2}}\right)\left(\delta_{i j}-\frac{k_{i} k_{j}}{k^{2}}\right) \\
& \times\left(G_{\mathbf{k}}-G_{\mathbf{k}^{\prime}}\right)\left\langle\mathbf{k}^{\prime} c|\Sigma| \mathbf{k} b\right\rangle,
\end{aligned}
$$

where $\mathbf{k}^{\prime}=\mathbf{k}+\mathbf{q}$. From Eqs. (4.8) $-(4.10)$ we note that the "non-Abelian" charge density in Eq. (4.10) is a correction of order $g^{2}$ relative to the "Abelian" one in Eq. (4.9). Thus, as anticipated after Eq. (4.2), it is consistent to evaluate this correction with the free kernel $G_{\mathbf{k}}=1 / 2 k$.

The integral in Eq. (4.10) is logarithmically ultraviolet divergent, so it must be evaluated with an upper cutoff. It turns out that this divergence is a part of the charge renormalization in QCD (see Sec. IV B below). To reconstruct the associated beta function, we need, as usual, only the coefficient of the divergent logarithm. The latter is insensitive to the details of the UV regularization, so we shall consider, for simplicity, a sharp momentum cutoff $\Lambda$.

Also, in order to isolate the leading logarithm, we can perform kinematical approximations relying on the inequality $k \gg q$ [since the external momentum $q$ is fixed, while the leading contribution to the integral in Eq. (4.10) comes from relatively large momenta]. Physically, we are indeed interested in smooth charge distributions. This allows us to re-

\footnotetext{
${ }^{4} \mathrm{By}$ which we mean transversality with respect to both $\mathbf{k}$ and $\mathbf{k}^{\prime}$, as requested by expressions (4.5) and (4.6).
} 
place $\mathbf{k}+\mathbf{q}$ by $\mathbf{k}$ and thus $\left(\delta_{i j}-k_{i}^{\prime} k_{j}^{\prime} / k^{\prime 2}\right)\left(\delta_{i j}-k_{i} k_{j} / k^{2}\right)$ by 2. By also using Eq. (4.8) for $\Sigma$, we then obtain

$$
\rho_{N A}^{a}(\mathbf{q})=\frac{C_{N}}{2} \omega^{a}(\mathbf{q}) X(\mathbf{q})
$$

where $C_{N}=\operatorname{Tr}\left(T^{3} T^{3}\right)=N$ for $S U(N)$, and

$$
X(\mathbf{q}) \equiv \int \frac{\mathrm{d}^{3} k}{(2 \pi)^{3}} \frac{\left[\varepsilon\left(\mathbf{k}^{\prime}\right)-\varepsilon(\mathbf{k})\right]^{2}}{\varepsilon(\mathbf{k}) \varepsilon\left(\mathbf{k}^{\prime}\right)\left[\varepsilon(\mathbf{k})+\varepsilon\left(\mathbf{k}^{\prime}\right)\right]},
$$

with $\varepsilon(\mathbf{k}) \equiv|\mathbf{k}|$. Here again we can replace $\mathbf{k}^{\prime} \simeq \mathbf{k}$ everywhere except in the numerator which must be expanded to second order in $q$ :

$$
[\varepsilon(\mathbf{k}+\mathbf{q})-\varepsilon(\mathbf{k})]^{2} \simeq q^{2} \cos ^{2} \theta
$$

where $\theta$ is the angle between the space vectors $\mathbf{k}$ and $\mathbf{q}$. The angular average yields $\left\langle\cos ^{2} \theta\right\rangle=\int_{0}^{\pi} d \theta \sin \theta \cos ^{2} \theta=2 / 3$, so, finally,

$$
X(\mathbf{q})=\frac{q^{2}}{12 \pi^{2}} \int \frac{\mathrm{d} k}{k},
$$

which is logarithmically divergent in the ultraviolet, as expected, but also in the infrared: the infrared divergence is an artifact of the previous manipulations (in a more careful calculation, this would be screened by $q$ ), and to the order of interest we can just regulate it with an ad hoc infrared cutoff $\mu$. This yields $\rho_{N A}^{a}=\alpha \rho_{A}^{a}$, with

$$
\alpha \equiv \frac{g^{2} C_{N}}{48 \pi^{2}} \ln \frac{\Lambda^{2}}{\mu^{2}} .
$$

The resulting value of the moment of inertia $\mathcal{I}_{a b} \equiv \delta \rho^{a} / \delta \omega^{b}$ reads, finally,

$$
\mathcal{I}_{a b}(\mathbf{q})=\frac{\delta_{a b}}{g^{2}} q^{2}(1+\alpha) .
$$

This should be compared to the corresponding Abelian result $^{5} I(q)=q^{2}$. We see that the quantum fluctuations in QCD produce an increase of the moment of inertia, which corresponds to the screening of color charges by quantum fluctuations. The size of the screening effect that we have obtained agrees indeed with the results of other approaches [25].

\footnotetext{
${ }^{5}$ The overall factor $1 / g^{2}$ in Eq. (4.16) is simply a consequence of our different normalizations for the field strengths in QCD and QED; compare, in this respect, Eqs. (3.12) and (4.1).
}

\section{B. Interaction energy in the presence of a background electric field}

Interesting properties of the vacuum include its response to an external chromo-electric field, which can be generated by an external constraint of the form

$$
H_{\mathrm{ext}}=g^{2} \int \mathrm{d}^{3} x \mathbf{E}_{\mathrm{ext}}^{a}(\mathbf{x}) \cdot \mathbf{E}^{a}(\mathbf{x}) .
$$

For this constraint, we shall compute the induced electric mean field and charge density, and the associated electrostatic energy. By comparing the latter with the bare Coulomb interaction, we shall then identify the chromo-electric susceptibility, or charge renormalization. As we shall see, the variational formalism provides a transparent picture of the underlying phenomena of screening and anti-screening.

The optimal state $\Psi_{c}$ in the presence of the constraint (4.17) is of the form

$$
\Psi_{c}[\mathbf{A}]=\mathcal{N}^{-1} \mathrm{e}^{-i\langle\mathbf{F} \mid \mathbf{A}\rangle} \exp \left\{-\left\langle A\left|\frac{G^{-1}}{4 g^{2}}\right| A\right\rangle\right\}
$$

where the parameter $F_{i}^{a}$ (the electric mean field) will be related to the external field $E_{\mathrm{ext}}^{i}$ by the variational equations [see Eq. (4.23) below]. Note that, in contrast to Eq. (4.3), there is no $\Sigma$ term in Eq. (4.18) above; this is so because the external perturbation here is different [compare Eqs. (4.17) and (3.33)]: it contains a term linear in $\mathbf{E}^{a}$, but no nonAbelian term like $\left[A^{i}, E^{i}\right]$. This situation is analogous to the case of an anharmonic oscillator with an external constraint $H_{e x t}=\alpha p$. The only changes are a factor $e^{i p_{0} x}$ and, in higher orders in $g$, a modification of the real part of the width.

According to the discussion in Sec. III [see especially Eqs. (3.37) and (3.38)], the energy functional to be minimized in this case is $\widetilde{E}=E_{c}-\left\langle H_{\mathrm{ext}}\right\rangle_{c}-\Delta \hat{E}_{T V}$. The terms involving $F_{a}^{i}$ in this functional read

$$
\begin{aligned}
E_{c}-E_{\mathrm{ext}}-\Delta \hat{E}_{T V}= & g^{2} \int \mathrm{d}^{3} x\left\{\frac{1}{2} \mathbf{F}^{a} \cdot \mathbf{F}^{a}-\mathbf{E}_{\text {ext }}^{a} \cdot \mathbf{F}^{a}\right\} \\
& -g^{2} f_{a c d} f_{b e f} \int \mathrm{d}^{3} x \mathrm{~d}^{3} y F_{i}^{d}(\mathbf{x}) F_{j}^{f}(\mathbf{y}) \\
& \times\left\langle\mathbf{x}\left|G_{i j}^{c e}\right| \mathbf{y}\right\rangle\left\langle a, \mathbf{x}\left|\frac{1}{2 \mathcal{I}}\right| b, \mathbf{y}\right\rangle
\end{aligned}
$$

The last term on the RHS corresponds to the ThoulessValatin correction, Eq. (3.38). Note that, because of the substraction of the average charge in $\hat{\mathcal{G}}^{a} \equiv \mathcal{G}^{a}-\left\langle\mathcal{G}^{a}\right\rangle_{c}$, it is only the non-Abelian part of the Gauss operator (i.e., the term $i\left[A^{i}, E^{i}\right]$ in Eq. (2.4)) which contributes to Eq. (4.19). To evaluate this contribution, we first rewrite it in momentum space: 


$$
\begin{aligned}
\Delta \hat{E}_{T V}= & g^{2} f_{a c d} f_{b e f} \int \frac{\mathrm{d}^{3} k}{(2 \pi)^{3}} \frac{\mathrm{d}^{3} q}{(2 \pi)^{3}} F_{i}^{d}(\mathbf{q}) F_{j}^{f}(-\mathbf{q}) G_{i j}^{c e}(\mathbf{k}) \\
& \times\left\langle a\left|\frac{1}{2 \mathcal{I}(\mathbf{k}+\mathbf{q})}\right| b\right\rangle .
\end{aligned}
$$

To the order of interest, we can replace the moment of inertia in the equation above by its leading order expression: $\mathcal{I}(\mathbf{k})$ $\simeq k^{2} / g^{2}$. Then, by performing similar manipulations as in the calculation of the $X$ term in Eqs. (4.10)-(4.14), we finally obtain

$$
\begin{aligned}
\Delta \hat{E}_{T V} & \simeq \frac{g^{4} C_{N}}{3} \int \frac{\mathrm{d}^{3} k}{(2 \pi)^{3}} \frac{\mathrm{d}^{3} q}{(2 \pi)^{3}} F_{i}^{a}(\mathbf{q}) F_{i}^{a}(-\mathbf{q}) \frac{1}{2 k} \frac{1}{k^{2}} \\
& \equiv \frac{g^{2} \delta}{2} \int \mathrm{d}^{3} x F_{i}^{a}(\mathbf{x}) F_{i}^{a}(\mathbf{x}),
\end{aligned}
$$

where

$$
\delta \equiv \frac{g^{2} C_{N}}{6 \pi^{2}} \int \frac{d k}{k}=\frac{g^{2} C_{N}}{12 \pi^{2}} \ln \left(\frac{\Lambda^{2}}{\mu^{2}}\right) .
$$

After inserting Eq. (4.21) into Eq. (4.19) and minimizing with respect to $F_{i}^{a}(\mathbf{x})$, we obtain

$$
(1-\delta) F_{a}^{i}(\mathbf{x})=E_{\operatorname{ext} a}^{i}(\mathbf{x}) .
$$

At this point it is convenient to introduce the charge distribution associated with the external field $E_{\mathrm{ext}}^{i}$,

$$
\rho_{\mathrm{ext}}^{a}(\mathbf{x}) \equiv \boldsymbol{\nabla} \cdot \mathbf{E}_{\mathrm{ext}}^{a}(\mathbf{x}),
$$

to be referred to as the external charge in what follows: this would be the charge in the system in the absence of polarization effects. The actual charge is rather

$$
\rho^{a}(\mathbf{x}) \equiv\left\langle\Psi_{c}\left|\mathcal{G}^{a}(\mathbf{x})\right| \Psi_{c}\right\rangle=\nabla \cdot \mathbf{F}^{a}=\frac{\rho_{\mathrm{ext}}^{a}}{1-\delta} \simeq \rho_{\mathrm{ext}}^{a}(1+\delta),
$$

where the second line follows from Eq. (4.24). Note that this relation implies an antiscreening of the external charge, since $\rho^{a}$ is bigger than $\rho_{\text {ext }}^{a}$. The difference $\rho-\rho_{\text {ext }}=\rho_{\text {ext }} \delta$ may be interpreted as an induced charge (see also Sec. IV C in Ref. [26] and Appendix A for an alternative computation of this quantity).

We are finally in position to compute the chromostatic interaction $E_{\text {chromo }}$ in the optimal state $\Psi_{c}$. This is given by Eq. (3.43) which, together with the above expressions (4.25) for $\left\langle\mathcal{G}^{a}(\mathbf{x})\right\rangle_{c}$, and (4.21) for $\Delta \hat{E}_{T V}$, implies

$$
E_{\text {chromo }} \simeq g^{2} \frac{1+3 \delta}{1+\alpha} \int \frac{\mathrm{d}^{3} q}{(2 \pi)^{3}} \rho_{\text {ext }}^{a}(\mathbf{q}) \rho_{\mathrm{ext}}^{a}(-\mathbf{q}) \frac{1}{2 \mathbf{q}^{2}},
$$

up to corrections of higher order in $g$. There is here a factor $(1+\delta)^{2} \simeq 1+2 \delta$ arising from the induced charge [cf. Eq. (4.25)], another one arising from the Thouless-Valatin cor- rection (4.21), and a factor $(1+\alpha)$ due to the moment of inertia [cf. Eq. (4.16)]. The interaction energy (4.26) is still Coulomb like,

$$
E_{\text {chromo }}=g_{R}^{2} \int \frac{\mathrm{d}^{3} q}{(2 \pi)^{3}} \rho_{\text {ext }}^{a}(\mathbf{q}) \rho_{\text {ext }}^{a}(-\mathbf{q}) \frac{1}{2 \mathbf{q}^{2}},
$$

but with a modified coupling constant given by

$$
g_{R}^{2}(\mu)=g^{2} \frac{1+3 \delta}{1+\alpha}
$$

or, to first order in $g^{2}$,

$$
\frac{1}{g_{R}^{2}(\mu)}=\frac{1}{g^{2}}-\frac{11 C_{N}}{48 \pi^{2}} \ln \frac{\Lambda^{2}}{\mu^{2}}
$$

This is the correct one-loop value for the renormalized coupling constant [25]. Note that, in the present calculation, this involves three types of contributions: indeed, the factor of 11 in the last equation has arisen as $11=8+4-1$, where the 8 corresponds to anti-screening by the induced charge [cf. Eq. (4.25)], the 4 is another anti-screening contribution due to the Thouless-Valatin correction $(4.21)$, and the $(-1)$ is a screening contribution arising via the correction of order $g^{2}$ to the moment of inertia.

\section{VACUUM ENERGY IN A MAGNETIC BACKGROUND FIELD}

In the previous section, we have studied the electric sector of the vacuum of Yang-Mills theory, by using a combination of variational and perturbative techniques. In what follows, we shall perform a similar analysis of the magnetic sector. To this aim, we consider Yang-Mills theory in the presence of a (constant) magnetic background field $\bar{B}_{a}^{i}$, and compute the background field energy by using the variational principle. The final result is not new (it coincides with the oneloop result by Savvidy [20]), but it rather serves as a test for our variational method in the magnetic sector and in the perturbative regime.

The relevant trial wave variational is the Gaussian functional $\Psi_{0}$ in Eq. (2.8) with the "center" field $\bar{A}_{a}^{i}(\mathbf{x})$ chosen so as to reproduce the desired magnetic field $\bar{B}_{a}^{i}$ (a convenient choice will be given later). This state is not gauge invariant, so its energy $E_{0} \equiv\left\langle\Psi_{0}|H| \Psi_{0}\right\rangle$, Eq. (2.9), must be corrected with the Thouless-Valatin energy $\Delta E_{T V}$, to be computed in the next subsection. Then, by applying the variational principle to the corrected energy $\widetilde{E}=E_{0}$ $-\Delta E_{T V}$, we shall determine the kernel of the Gaussian (in Sec. V B). Finally, in Sec. V C, we shall compute the energy of the magnetic field and the associated gluon condensate, and verify that these quantities are related by the trace anomaly relation, as they should. In this calculation, the standard one-loop beta function will emerge once again. 


\section{A. The Thouless-Valatin energy in the background field}

We start by computing the moment of inertia $\mathcal{I}_{a b}(\mathbf{x}, \mathbf{y})$ in the presence of the background field $\bar{A}_{a}^{i}(\mathbf{x})$. As already explained, this requires constructing the variational ground state $\Psi_{\omega}$ for the constrained Hamiltonian $H_{\omega}$ in Eq. (3.33). This state is of the form [compare to Eq. (4.3)]

$$
\Psi_{\omega}=\mathcal{N}^{-1} e^{-i\langle F \mid A-\bar{A}\rangle} \exp \left\{-\left\langle A-\bar{A}\left|\frac{G^{-1}}{4 g^{2}}-i \Sigma\right| A-\bar{A}\right\rangle\right\},
$$

where the parameters $F_{a}^{i}$ and $\sum_{i j}^{a b}$ are related to $\omega^{a}$ by the variational principle (recall the discussion in Sec. IV A). It turns out that the matrix $\Sigma$ will not play any role in what follows: indeed, below we shall need the moment of inertia only to leading order in $g$, while $\Sigma$ counts starting with order $g^{2}$ (cf. Sec. IV A). We then write, as in Eq. (4.4),

$$
E_{\omega}=\langle H\rangle_{\omega}-\int d^{3} x \omega^{a}(\mathbf{x})\left\langle\mathcal{G}^{a}(\mathbf{x})\right\rangle_{\omega}
$$

with $\langle H\rangle_{\omega}$ given by Eq. (4.5), and

$$
\left\langle\mathcal{G}^{a}(\mathbf{x})\right\rangle_{\omega}=\left(\mathcal{D}_{i} F^{i}\right)^{a}(\mathbf{x})+\mathcal{O}\left(g^{2}\right),
$$

where $\mathcal{D}^{i} \equiv \partial^{i}-i \bar{A}^{i}$ is the covariant derivative defined by the background field [cf. Eq. (2.11)], and the neglected terms, of $\mathcal{O}\left(g^{2}\right)$, would involve $\Sigma$ [cf. Eq. (4.6)]. The variation with respect to $F_{a}^{i}$ yields then

$$
F_{a}^{i}(\mathbf{x})=-\frac{1}{g}\left(\mathcal{D}_{i} \omega\right)^{a}(\mathbf{x})
$$

which differs from the corresponding expression in Eq. (4.7) only by the replacement of the ordinary derivative $\partial_{i}$ by the covariant one $\mathcal{D}_{i}$. Together with Eq. (5.3), this provides the moment of inertia to the order of interest:

$$
\begin{aligned}
\mathcal{I}^{a b}(\mathbf{x}, \mathbf{y}) & \equiv \frac{\delta\left\langle\mathcal{G}^{a}(\mathbf{x})\right\rangle_{\omega}}{\delta \omega^{b}(\mathbf{y})}=-\frac{1}{g^{2}}\left(\mathcal{D}_{x}^{2}\right)^{a b} \delta^{(3)}(\mathbf{x}-\mathbf{y})+\mathcal{O}(1) \\
& =\frac{1}{g^{2}}\left\langle a, \mathbf{x}\left|\Pi^{2}\right| b, \mathbf{y}\right\rangle+\mathcal{O}(1)
\end{aligned}
$$

We have introduced here the kinetic momentum $\Pi_{j} \equiv i \mathcal{D}_{j}$ $=i \delta_{j}+\bar{A}_{j}$ and $\Pi^{2} \equiv \Pi_{j} \Pi_{j}$.

Within the same accuracy, one has also

$$
\begin{aligned}
\left\langle\Psi_{0}\left|\mathcal{G}^{a}(\mathbf{x}) \mathcal{G}^{b}(\mathbf{y})\right| \Psi_{0}\right\rangle & \approx \frac{1}{4 g^{2}} \mathcal{D}_{i, \mathbf{x}}^{a c} \mathcal{D}_{d b}^{j, \mathbf{y}}\left(G^{-1}\right)_{i j}^{c d}(\mathbf{x}, \mathbf{y}) \\
& =\frac{1}{4 g^{2}}\left\langle a, \mathbf{x}\left|\Pi_{i} G_{i j}^{-1} \Pi_{j}\right| b, \mathbf{y}\right\rangle .
\end{aligned}
$$

We are now in position to compute the Thouless-Valatin energy $\Delta E_{T V}[$ cf. Eq. (3.34)] by combining Eqs. (5.5) and (5.6), one obtains

$$
\Delta E_{T V} \approx \frac{1}{8} \int d^{3} x\left\langle a, \mathbf{x}\left|\left(\Pi_{i} \frac{1}{\Pi^{2}} \Pi_{j}\right) G_{i j}^{-1}\right| a, \mathbf{x}\right\rangle,
$$

up to corrections of order $g^{2}$.

\section{B. Variational equation for $G$}

The improved energy functional $\widetilde{E}=E_{0}-\Delta E_{T V}$ reads therefore [cf. Eqs. (2.9) and (5.7)]

$$
\begin{aligned}
\widetilde{E}= & \int d^{3} \mathbf{x}\left\{\frac{1}{2 g^{2}} \bar{B}_{i}^{a}(\mathbf{x}) \bar{B}_{i}^{a}(\mathbf{x})\right. \\
& +\frac{1}{8}\left\langle a, \mathbf{x}\left|\left(\delta_{i j}-\Pi_{i} \frac{1}{\Pi^{2}} \Pi_{j}\right) G_{i j}^{-1}\right| a, \mathbf{x}\right\rangle \\
& \left.+\frac{1}{2} \operatorname{Tr}[K G(\mathbf{x}, \mathbf{x})]+\mathcal{O}\left(g^{2}\right)\right\} .
\end{aligned}
$$

Note that the last two terms in Eq. (2.9) do not contribute to this order. As obvious from this equation, the ThoulessValatin correction makes the kinetical part of the energy covariantly transverse. Since the operator $K_{i j}$ is transverse as well [cf. Eq. (2.10)],

$$
K_{i j}=\Pi^{2} \delta_{i j}-\Pi_{i} \Pi_{j}+2\left[\Pi_{i}, \Pi_{j}\right]
$$

it follows that the projected energy (5.8) involves only the transverse components of the kernel $G$. Thus, without loss of generality, we can restrict ourselves to a (covariantly) transverse kernel in what follows:

$$
\Pi^{i} G_{i j}^{-1}=0=G_{i j}^{-1} \Pi^{j} .
$$

To formalize this, it is convenient to introduce transverse and longitudinal projectors as follows:

$$
\hat{P}_{i j} \equiv \Pi_{i} \frac{1}{\Pi^{2}} \Pi_{j}, \quad \hat{Q} \equiv 1-\hat{P}
$$

They satisfy

$$
\begin{aligned}
& \hat{P}_{i j}^{2}=\Pi_{i} \frac{1}{\Pi^{2}} \Pi_{k} \Pi^{k} \frac{1}{\Pi^{2}} \Pi^{j}=\hat{P}_{i j}, \\
& \hat{Q}_{i j}^{2}=(1-\hat{P})_{i j}^{2}=(1-\hat{P})_{i j}=\hat{Q}_{i j} .
\end{aligned}
$$

Then, a transverse kernel is one satisfying $G=\hat{Q} G \hat{Q}$ (and similarly for $G^{-1}$ ). For such a kernel, the variational principle [i.e., the minimization of $\widetilde{E}$, Eq. (5.8), with respect to $G]$ produces the following gap equation:

$$
\frac{1}{4 G^{2}} \simeq K
$$


which determines $G$ to the order of interest. In particular, as $\bar{A} \rightarrow 0, \quad G$ reduces to the free, or Abelian, expression in Eqs. (4.2) and (3.27). Thus, the only non-trivial effects which are taken here into account are those associated with the background field.

Note that Eq. (5.13) can only be valid at sufficiently high energy or small coupling constant: indeed, the operator $K$ admits negative modes $[20,27,28]$. We thus assume that an infrared cutoff has been set-this does not affect the ultraviolet behavior of the theory, which is our main interest here.

\section{Energy of the background field}

The previous equations provide the optimal Gaussian kernel for a given background field $\bar{A}$ and thus the effective potential $V(\bar{A})$ which is the expectation value of the energy in this state. The next step in our variational approach is to find the minimum of the effective potential. Constructing $V$ for an arbitrary background is however a difficult task. For this reason we now consider a restricted variational space defined by the following background field:

$$
\bar{A}_{x}=0, \quad \bar{A}_{y}=x B T^{3}, \quad \bar{A}_{z}=0 .
$$

This corresponds to a constant magnetic field in the $z$ direction and in the third color.

With this choice of the background field, we are now able to compute the energy (5.8) in the optimal variational state, which is the Gaussian state (2.8) with a transverse [in the sense of Eq. (5.10)] kernel $G^{-1}$ satisfying Eq. (5.13). The latter equation shows that, at the minimum, the following identity holds:

$$
\operatorname{Tr}\{K\langle\mathbf{x}|G| \mathbf{x}\rangle\}=\frac{1}{4} \operatorname{Tr}\left\langle\mathbf{x}\left|G^{-1}\right| \mathbf{x}\right\rangle .
$$

That is, magnetic and electric fluctuations have equal energies in our variational ground states, which is merely the virial theorem in the present context. Thus,

$$
\widetilde{E}_{\text {min }} \simeq \int d^{3} \mathbf{x}\left\{\frac{1}{2 g^{2}} \bar{B}_{i}^{a}(\mathbf{x}) \bar{B}_{i}^{a}(\mathbf{x})+\frac{1}{4}\left\langle a, \mathbf{x}\left|G^{-1}\right| a, \mathbf{x}\right\rangle\right\}
$$

This involves the matrix element $\left\langle\mathbf{x}\left|G^{-1}\right| \mathbf{x}\right\rangle$, which we shall compute in Appendix A by using the Schwinger proper-time representation [cf. Eq. (5.13)]:

$$
\left\langle\mathbf{x}\left|G^{-1}\right| \mathbf{x}\right\rangle=\frac{1}{\sqrt{\pi}} \int_{0}^{\infty} \frac{d t}{t^{3 / 2}}\left\langle\mathbf{x}\left|\left(1-e^{-t K}\right)\right| \mathbf{x}\right\rangle .
$$

This integral develops ultraviolet divergences as $t \rightarrow 0$, which we shall regularize by shifting the lower bounds of the integral from 0 to $1 / \Lambda^{2}$. As in the electric case, we are mainly interested in the ultraviolet renormalization of the energy (5.16); to this aim, it is sufficient to extract the terms which diverge when $\Lambda \rightarrow \infty$ in Eq. (5.17). This is described in detail in Appendix A, from which we quote here the final result:

$$
\operatorname{Tr}\left\langle\mathbf{x}\left|G^{-1}\right| \mathbf{x}\right\rangle=(\cdots) \Lambda^{4}-\frac{11 C_{N}}{48 \pi^{2}} B^{2} \ln \frac{\Lambda^{2}}{B}+\mathcal{O}\left(g^{2}\right)
$$

(the coefficient in front of $\Lambda^{4}$ is an uninteresting fieldindependent number that will be omitted in what follows). Remarkably, there is no divergent term in $\Lambda^{2}$ (which, for dimensional reasons, would be necessarily of the form $\left.\Lambda^{2} B\right)$ : this is so because of rotational and gauge symmetries which require the magnetic field to enter only in the scalar product $\mathbf{S} \cdot \mathbf{B}_{a} T^{a}=B S_{z} T^{3}$, whose trace is however zero (see Appendix A for more details).

Note also the numerical factor in front of the logarithmic divergence in Eq. (5.18): this is the factor leading to the correct one-loop beta function after renormalization (see below). The projection on (covariantly) transverse Gaussian states has been crucial in getting this factor right: without this, we would have obtained a factor of $\frac{7}{2}$ instead of the correct factor of $\frac{11}{3}$ [compare in this respect Eqs. (B19) and (B20) in Appendix A].

Finally note that the field strength $B$ appears as an infrared cutoff in Eq. (5.18). This is expected from Eqs. (B6) and (B7) where the proper time variable always appears in the combination $t B$. However, a complete derivation of Eq. (5.18) requires a detailed treatment of unstable modes.

To conclude,

$$
\widetilde{E} / V \simeq \frac{1}{2 g^{2}} B^{2}-C_{N} \frac{11}{48 \pi^{2}} \frac{B^{2}}{2} \ln \frac{\Lambda^{2}}{B},
$$

showing that the background field energy has no field dependent UV divergences other than the logarithmic one which can be absorbed into the renormalization of the coupling constant. We then write, as usual ( $\mu$ is the substraction scale),

$$
\frac{1}{g_{R}^{2}(\mu)}=\frac{1}{g^{2}}-C_{N} \frac{11}{48 \pi^{2}} \ln \frac{\Lambda^{2}}{\mu^{2}}
$$

which provides the correct one-loop beta function, as anticipated. The renormalized field energy density reads then

$$
\mathcal{H}(B)=\frac{B^{2}}{2 g_{R}^{2}(\mu)}+\frac{B^{2}}{2} \frac{11}{48 \pi^{2}} C_{N} \ln \frac{B}{\mu^{2}},
$$

which coincides with the result obtained by Savvidy in perturbation theory [20]. An advantage of the present approach, however, is that it can be improved by using a larger variational space, which is expected to cure the difficulties associated with negative modes [27,28].

As discussed in [20], the energy density (5.21) exhibits a minimum for a non-zero value $B=B_{\text {min }}$ of the background field, with

$$
B_{\min }=\frac{\mu^{2}}{\sqrt{e}} \exp \left(-\frac{16 \pi^{2}}{g_{R}^{2}(\mu)} \frac{3}{11 C_{N}}\right) .
$$


The value of the energy density at this minimum is

$$
\langle\mathcal{H}\rangle_{\text {min }}=-\frac{1}{64 \pi^{2}} \frac{11}{3} C_{N} B_{\text {min }}^{2}
$$

which is indeed negative. Our variational vacuum state is therefore characterized by a magnetic field condensate (see, however, Refs. [27,28] for potential problems with such a state).

From the previous results, it is now straightforward to evaluate the gluon condensate in our variational vacuum:

$$
\begin{aligned}
\left\langle F^{\mu \nu} F_{\mu \nu}\right\rangle & \equiv 2 g^{2}\left(\frac{1}{g^{2}}\left\langle B_{i}^{a} B_{i}^{a}\right\rangle-\left\langle g^{2} E_{i}^{a} E_{i}^{a}\right\rangle\right) \\
& =2 B_{\text {min }}^{2},
\end{aligned}
$$

where the second line follows from the aforementioned "virial theorem"' (5.15). Equations (5.23) and (5.24) can be combined into

$$
\langle\mathcal{H}\rangle_{\text {min }}=-\frac{11 C_{N}}{128 \pi^{2}}\left\langle F^{\mu \nu} F_{\mu \nu}\right\rangle_{\text {min }}
$$

which is consistent, as it should, with the trace anomaly relation

$$
\left\langle\theta_{\mu}^{\mu}\right\rangle=\frac{\beta(g)}{2 g^{3}}\left\langle F^{\mu \nu} F_{\mu \nu}\right\rangle .
$$

Indeed, with $\langle\mathcal{H}\rangle=\frac{1}{4}\left\langle\theta_{\mu}^{\mu}\right\rangle$ and the one-loop beta function [which here is a consequence of Eq. (5.20)]

$$
\beta(g)=-\frac{11}{48 \pi^{2}} C_{N} g^{3},
$$

Eq. (5.26) becomes identical to Eq. (5.25).

An attractive feature of the formula (5.25) is that it involves two quantities which are independently accessible experimentally (at least indirectly). Indeed, the left-hand side of this equation is the energy density of the vacuum, which can be identified with the fourth power of the bag constant, $\mathcal{B}^{4}=-(240 \mathrm{MeV})^{4}[29]$, whereas the right-hand side depends on the gluon condensate which is known from Ref. [30] to be $0.5 \mathrm{GeV}^{4}$. These values are compatible with Eq. (5.25) within a $20 \%$ accuracy.

\section{CONCLUSION}

In this paper we have proposed an improved energy functional for variational calculations in gauge field theories. This functional contains a nonlocal term which approximately corresponds to the energy gain when projecting on gauge invariant states. This allows one to use Gaussian states as trial functionals and thus perform analytic calculations for physical observables such as the chromoelectric and chromomagnetic susceptibilities, energy expectation values and the gluon condensate.

The main purpose of this work was to check the ultraviolet behavior of our approximation scheme. By performing variational calculations near the perturbative vacuum we have shown that divergences can be eliminated by a renormalization of the coupling constant. This has allowed us to recover the familiar one loop beta function in a way which makes transparent the various screening and antiscreening contributions. In particular the screening term arises naturally in our formalism, which was not the case in earlier variational approaches [15]. We have also tested our variational method in the magnetic sector, checking that it reproduces the one-loop result by Savvidy [20] for the background field energy. This calculation provides us with another derivation of the one-loop beta function.

Thus, our formalism appears to correctly reproduce the expected behavior of non-Abelian theories in the ultraviolet sector. This strongly encourages us to study its predictions in the nonperturbative regime. Indeed, as a variational approach, it is not at all restricted to the vicinity of the perturbative ground state or to small values of the coupling constant. We would like to also emphasize that the ThoulessValatin correction is the first step in an approximation scheme which can be constructed systematically. Indeed, it is the first order term [23] in boson expansion methods which have been constructed by Schwinger [31], Dyson [32], Holstein and Primakoff [33], and Blaizot and Marshalek [34].

In QCD, boson expansions could be formulated in a gauge invariant way by exploiting the methods developed in Ref. [10]. There, it has been shown how to construct a gauge invariant wave functional by solving Ward identities within the so-called "gauge technique." The resulting functional is nonlinear to all orders in the gauge potentials and could be used as the starting point of a boson expansion formalism. Specifically one could imagine constructing new boson operators in such a way that the wave functional becomes a Gaussian in the new representation. A priori these new operators would themselves be expressed as an infinite series in the original field operators. One could expect that the first few terms in such an expansion would generate, in the energy functional, the Thouless-Valatin correction discussed in the present paper. Exploring this approach would then not only provide a derivation of our present formula, but also a systematic construction of the higher order terms.

Let us finally mention some physical situations where the variational approach could be useful. The instabilities occurring in calculations using a constant magnetic background field $[27,28]$ (cf. Sec. V) could be dealt with by properly expanding the variational space so as to include the subspace spanned by the unstable modes. More generally, this variational approach could give us some insight into the nonpertubative infrared physics related to confinement or the generation of mass scales in QCD [18]. Sum rules and the gluon condensate at finite temperature appear to be another promising field of investigation.

\section{ACKNOWLEDGMENTS}

We are most grateful to A. K. Kerman for valuable remarks, regarding in particular the physical significance of the moment of inertia. Stimulating discussions with J. Polonyi and L. McLerran are also gratefully acknowledged. 


\section{APPENDIX A: PHYSICAL INTERPRETATION OF SEC. IV B IN TERMS OF THE INDUCED CHARGE}

Let us show another interpretation (and computation) of the relation between $\rho^{a}$ and the external charge. What follows is directly inspired from Gottfried and Weisskopf in Sec. IV.C of [26]. Let us assume that the system is in the presence of a given distribution of external charges $\rho_{\text {ext }}^{a}(\mathbf{x})$ $=\boldsymbol{\nabla} \cdot \mathbf{E}_{\text {ext }}^{a}(\mathbf{x})$ and compute the corresponding induced charge created by the quantum fluctuations of the gauge field; it is given by the mean value $\left\langle-f_{a b c} A_{i}^{b}(\mathbf{x})\left(E_{L}^{i}\right)^{c}(\mathbf{x})\right\rangle$ where $\mathbf{E}_{L}$ is the longitudinal part of the chromoelectric field operator $\mathbf{E}$ [26]. This operator is a non-dynamical variable which is fixed by Gauss' law:

$$
\boldsymbol{\nabla} \cdot \mathbf{E}_{L}^{a}(\mathbf{x})=\rho_{\text {ext }}(\mathbf{x})-f_{a b c} A_{i}^{b}(\mathbf{x})\left(E_{L}^{i}\right)^{c}(\mathbf{x})
$$

which can be solved perturbatively, setting $\mathbf{E}_{L}^{a}(\mathbf{x})$ $=\mathbf{E}_{L}^{a(0)}(\mathbf{x})+\mathbf{E}_{L}^{a(1)}(\mathbf{x})+\mathbf{E}_{L}^{a(2)}(\mathbf{x})+\cdots$. In fact, only the first two terms will be needed for a development of the total charge in first order of $g^{2}$. They verify the following set of equations:

$$
\begin{aligned}
& \boldsymbol{\nabla} \cdot \mathbf{E}_{L}^{(0) a}(\mathbf{x})=\rho_{\text {ext }}^{a}(\mathbf{x}), \\
& \boldsymbol{\nabla} \cdot \mathbf{E}_{L}^{(1) a}(\mathbf{x})=-f_{a b c} A_{i}^{b}(\mathbf{x})\left(E_{L}^{i}\right)^{(0) c}(\mathbf{x}) .
\end{aligned}
$$

The first equation shows that $\mathbf{E}_{L}^{(0)}(\mathbf{x})=\mathbf{E}_{\text {ext }}(\mathbf{x})$. Then the second equation is solved by

$$
\left(E_{L}^{i}\right)^{(1) c}(\mathbf{x})=-\int \frac{\mathrm{d}^{3} y}{4 \pi} \frac{x_{i}-y_{i}}{|\mathbf{x}-\mathbf{y}|^{3}} f_{c d e} A_{j}^{d}(\mathbf{y})\left(E_{e x t}^{j}\right)^{e}(\mathbf{y}) .
$$

The total charge reads therefore

$$
\begin{aligned}
\rho_{\text {tot }}^{a}(\mathbf{x})= & \left\langle\mathcal{G}^{a}(\mathbf{x})\right\rangle \\
= & \rho_{\text {ext }}^{a}(\mathbf{x})+\left\langle-f_{a b c} A_{i}^{b}(\mathbf{x})\left(E_{\text {ext }}^{i}\right)^{c}(\mathbf{x})\right\rangle \\
& +\left\langle-f_{a b c} A_{i}^{b}(\mathbf{x})\left(E_{L}^{i}\right)^{(1) c}(\mathbf{x})\right\rangle \\
\equiv & \rho_{\text {ext }}^{a}(\mathbf{x})+\rho_{\text {ind }}^{a}(\mathbf{x}) .
\end{aligned}
$$

The first term in $\rho_{\text {ind }}^{a}$ vanishes since linear in $A_{i}$, while the second term yields

$$
\begin{aligned}
\rho_{\text {ind }}^{a}(\mathbf{x}) & \equiv\left\langle-f_{a b c} A_{i}^{b}(\mathbf{x})\left(E_{L}^{i}\right)^{(1) c}(\mathbf{x})\right\rangle_{c} \\
& =f_{a b c} f_{c d e} \int \frac{\mathrm{d}^{3} y}{4 \pi} \frac{x_{i}-y_{i}}{|\mathbf{x}-\mathbf{y}|^{3}} G_{i j}^{b d}(\mathbf{x}-\mathbf{y})\left(E_{\text {ext }}\right)_{j}^{e}(\mathbf{y})
\end{aligned}
$$

or, after a Fourier transform,

$$
\rho_{i n d}^{a}(\mathbf{q})=f_{a b c} f_{c d e}\left(E_{e x t}\right)_{j}^{e}(\mathbf{q}) \int \frac{\mathrm{d}^{3} x}{4 \pi} e^{-i \mathbf{q} \cdot \mathbf{x}} \frac{x_{i}}{|\mathbf{x}|^{3}} G_{i j}^{b d}(\mathbf{x}) .
$$

By also using

$$
\int \frac{\mathrm{d}^{3} x}{4 \pi} e^{i \mathbf{k} \cdot \mathbf{x}} \frac{x_{i}}{|\mathbf{x}|^{3}}=i \frac{k_{i}}{\mathbf{k}^{2}}
$$

we finally deduce

$$
\rho_{i n d}^{a}(\mathbf{q})=f_{a b c} f_{c d e} i\left(E_{e x t}\right)_{j}^{e}(\mathbf{q}) \int \frac{\mathrm{d}^{3} k}{(2 \pi)^{3}} \frac{(k-q)_{i}}{|\mathbf{k}+\mathbf{q}|^{2}} G_{i j}^{b d}(\mathbf{k}) .
$$

Since $G$ is transverse, this expression reduces to

$$
\rho_{i n d}^{a}(\mathbf{q})=-f_{a b c} f_{c d e} i\left(E_{e x t}\right)_{j}^{e}(\mathbf{q}) \int \frac{\mathrm{d}^{3} k}{(2 \pi)^{3}} \frac{q_{i}}{|\mathbf{k}+\mathbf{q}|^{2}} v G_{i j}^{b d}(\mathbf{k}) \text {. }
$$

Furthermore, for a smooth charge distribution we can approximate $\mathbf{k}+\mathbf{q}$ by $\mathbf{k}$ in the above integral. Noting that the result vanishes unless $i=j$ we have

$$
\rho_{\text {ind }}^{a}(\mathbf{q})=\gamma \rho_{\text {ext }}^{a}(\mathbf{q}),
$$

with

$$
\gamma=g^{2} C_{N} \frac{1}{6 \pi^{2}} \int_{0}^{\infty} \frac{\mathrm{d} k}{\varepsilon(\mathbf{k})}=\delta
$$

The total charge is then given by

$$
\begin{aligned}
\rho_{\text {tot }}^{a}(\mathbf{q}) & =\rho_{\text {ext }}^{a}(\mathbf{q})+\rho_{\text {ind }}^{a}(\mathbf{q}) \\
& =\rho_{\text {ext }}^{a}(\mathbf{q})(1+\delta),
\end{aligned}
$$

which is precisely the expression obtained in Sec. IV B [cf. Eq. (4.25)].

\section{APPENDIX B: PROPER-TIME CALCULATION OF THE ENERGY DENSITY}

Let us present here in some detail the calculation of the quantity $\operatorname{Tr} G^{-1}$ which enters the energy of the magnetic field in Sec. V C. According to the Schwinger proper-time representation (5.17), one needs the matrix element $\left\langle\mathbf{x}\left|e^{-t K}\right| \mathbf{x}\right\rangle$. Since, moreover, we are mainly interested in the ultraviolet behavior of the energy, this expression is needed only at small values of $t$, which allows us to perform expansions in powers of $t$ whenever necessary.

As explained in Sec. V C, we shall use the background field in Eq. (5.14) for which

$$
\left[\mathcal{D}_{i}, \mathcal{D}_{j}\right]^{a b}=-f^{a b 3} \epsilon^{3 i j} B=T^{3} S_{z} B,
$$

and therefore $\left[\Pi_{i}, \Pi_{j}\right]^{a b}=-\left[\mathcal{D}_{i}, \mathcal{D}_{j}\right]^{a b}=-\left(T^{3}\right)_{a b}\left(S_{z}\right)_{i j} B$. It is convenient to define the operator [cf. Eq. (5.9)]

$$
\widetilde{K}_{i j} \equiv K_{i j}+\Pi_{i} \Pi_{j}=\Pi^{2} \delta_{i j}+2\left[\Pi_{i}, \Pi_{j}\right],
$$

in terms of which $K_{i j}$ can be rewritten as follows:

$$
K=\widetilde{K} \hat{Q}
$$

By also using $[\widetilde{K}, \hat{Q}]=0$, and $\hat{Q}+\hat{P}=1$, we deduce 


$$
e^{-t K}=e^{-t \tilde{K}} \hat{Q}+\hat{P} .
$$

We thus have to compute the matrix element $\left\langle\mathbf{x}\left|e^{-t \tilde{K}} \hat{Q}\right| \mathbf{x}\right\rangle$, with

$$
\begin{gathered}
\widetilde{K}=\Pi^{2}-2 T^{3} S_{z} B \\
\left\langle\mathbf{x}\left|e^{-t \tilde{K}}\right| \mathbf{x}\right\rangle=\left\langle\mathbf{x}\left|e^{-t \Pi^{2}} e^{2 t T^{3} S_{z} B}\right| \mathbf{x}\right\rangle,
\end{gathered}
$$

where the second line follows since $\left[\Pi^{2}, T^{3} S_{z}\right]=0$.

The computation of $\left\langle\mathbf{x}\left|e^{-t \Pi^{2}}\right| \mathbf{x}\right\rangle$ is well known in the literature [35], with the result

$$
\left\langle\mathbf{x}\left|e^{-t \Pi^{2}}\right| \mathbf{x}\right\rangle=\left(\frac{1}{4 \pi t}\right)^{3 / 2} \frac{t T^{3} B}{\sinh \left(t T^{3} B\right)} .
$$

We thus obtain:

$$
\left\langle\mathbf{x}\left|e^{-t \widetilde{K}}\right| \mathbf{x}\right\rangle=\left(\frac{1}{4 \pi t}\right)^{3 / 2} \frac{t T^{3} B}{\sinh \left(t T^{3} B\right)} e^{2 t T^{3} S_{z} B} .
$$

By also using $\hat{Q}=1-\hat{P}$ and $\Pi_{i} \Pi^{2}=\widetilde{K} \Pi_{i}$, one then rewrites $\left\langle\mathbf{x}\left|e^{-t \tilde{K}} \hat{Q}\right| \mathbf{x}\right\rangle$ as

$$
\left\langle\mathbf{x}\left|e^{-t \tilde{K}}\right| \mathbf{x}\right\rangle-\left\langle\mathbf{x}\left|e^{-t \widetilde{K}} \Pi_{i} \Pi_{j} \frac{1}{\widetilde{K}}\right| \mathbf{x}\right\rangle .
$$

The last term of this equation can be obtained from $\left\langle\mathbf{x}\left|e^{-t \widetilde{K}} \Pi_{i} \Pi_{j}\right| \mathbf{x}\right\rangle$ by integration over $t$.

To calculate $\left\langle\mathbf{x}\left|e^{-t \widetilde{K}} \Pi_{i} \Pi_{j}\right| \mathbf{x}\right\rangle$, we follow Schwinger's method [36]: We work in Heisenberg's representation with $t=i s$ and deduce

$$
\begin{aligned}
\left\langle\mathbf{x}\left|e^{-t \Pi^{2}} \Pi_{i} \Pi_{j}\right| \mathbf{x}\right\rangle & =\left\langle\mathbf{x}\left|e^{-i s \Pi^{2}} \Pi_{i} \Pi_{j}\right| \mathbf{x}\right\rangle \\
& =\left\langle\mathbf{x}(s)\left|\Pi_{i}(0) \Pi_{j}(0)\right| \mathbf{x}(0)\right\rangle
\end{aligned}
$$

where

$$
\begin{aligned}
& x_{i}(s)=e^{i s \Pi^{2}} x_{i}(0) e^{-i s \Pi^{2}}, \\
& \Pi(s)=e^{i s \Pi^{2}} \Pi(0) e^{-i s \Pi^{2}} .
\end{aligned}
$$

The operator $U(s)=e^{-i s \Pi^{2}}$ can be interpreted as the evolution operator of a particle governed by the Hamiltonian $\Pi^{2}$. We have

$$
\begin{aligned}
\frac{d x_{i}}{d s} & =i\left[\Pi^{2}, x_{i}\right](s) \\
& =2 \Pi(s)
\end{aligned}
$$

and

$$
\frac{d \Pi}{d s}=i\left[\Pi^{2}, \Pi\right](s) .
$$

Using $\left[\Pi^{2}, \Pi\right](s)=2\left[\Pi_{k}, \Pi_{i}\right] \Pi^{k}=-2 i \mathcal{F}_{i k} \Pi^{k}$, where

$$
\mathcal{F}_{i j}^{a b} \equiv i\left[\mathcal{D}_{i}, \mathcal{D}_{j}\right]^{a b}=i T^{3} S_{z} B,
$$

one obtains $\Pi(s)=\left(e^{2 s \mathcal{F}}\right)_{i k} \Pi^{k}(0)$, and thus

$$
\begin{aligned}
x_{i}(s)-x_{i}(0) & =\left(\frac{e^{2 s \mathcal{F}}-1}{\mathcal{F}}\right)_{i j} \Pi^{j}(0) \\
& \equiv R_{i j}^{-1} \Pi^{j}(0) .
\end{aligned}
$$

The matrix element $\left\langle\mathbf{x}\left|e^{-t \Pi^{2}} \Pi_{i} \Pi_{j}\right| \mathbf{x}\right\rangle$ can be now computed as [cf. Eq. (B9)]

$$
\begin{aligned}
\left\langle\mathbf{x}(s)\left|\Pi_{i}(0) \Pi_{j}(0)\right| \mathbf{x}(0)\right\rangle & =\left\langle\mathbf{x}(s)\left|R_{i m} R_{j n}\left[x_{m}(s)-x_{m}(0)\right]\left[x_{n}(s)-x_{n}(0)\right]\right| \mathbf{x}(0)\right\rangle \\
& =R_{i m} R_{j n}\left\langle\mathbf{x}(s)\left|x_{m}(s) x_{n}(s)-x_{m}(s) x_{n}(0)-x_{n}(s) x_{m}(0)+x_{m}(0) x_{n}(0)+\left[x_{n}(s), x_{m}(0)\right]\right| \mathbf{x}(0)\right\rangle \\
& =R_{i m} R_{j n} R_{n k}^{-1}\left[\Pi_{k}(0), x_{m}(0)\right]\langle\mathbf{x}(s) \mid \mathbf{x}(0)\rangle \\
& =-i R_{i j}\langle\mathbf{x}(s) \mid \mathbf{x}(0)\rangle .
\end{aligned}
$$

Returning to the variable $s=-i t$, we obtain

$$
\left\langle\mathbf{x}\left|e^{-t \Pi^{2}} \Pi_{i} \Pi_{j}\right| \mathbf{x}\right\rangle=\left(\frac{-i \mathcal{F}}{e^{-2 i t \mathcal{F}}-1}\right)_{i j}\left\langle\mathbf{x}\left|e^{-t \Pi^{2}}\right| \mathbf{x}\right\rangle,
$$

and thus, finally,

$$
\begin{aligned}
\left\langle\mathbf{x}\left|e^{-t \tilde{K}} \Pi_{i} \Pi_{j}\right| \mathbf{x}\right\rangle & =\frac{T^{3} S_{z} B}{2 \sinh \left(t T^{3} S_{z} B\right)}\left(\frac{1}{4 \pi t}\right)^{3 / 2} \frac{t T^{3} B}{\sinh \left(t T^{3} B\right)} e^{t T^{3} S_{z} B} \\
& =\frac{1}{2 t}\left(\frac{1}{4 \pi t}\right)^{3 / 2}\left\{1+T^{3} B S_{z} t+\left(\frac{\left(T^{3}\right)^{2} B^{2} S_{z}^{2}}{3}-\frac{\left(T^{3}\right)^{2} B^{2}}{6}\right) t^{2}+\cdots\right\}
\end{aligned}
$$


where in writing the second line we have developed up to the second order in $t$.

The last equation can be now integrated over $t$ to obtain the second term in Eq. (B8):

$$
\begin{aligned}
\left\langle\mathbf{x}\left|e^{-t \tilde{K}} \hat{P}\right| \mathbf{x}\right\rangle= & \left(\frac{1}{4 \pi t}\right)^{3 / 2}\left\{\frac{1}{3}+T^{3} B S_{z} t\right. \\
& \left.+\left(\frac{\left(T^{3}\right)^{2} B^{2}}{6}-\frac{\left(T^{3}\right)^{2} B^{2} S_{z}^{2}}{3}\right) t^{2}+\cdots\right\} .
\end{aligned}
$$

Developing $\left\langle\mathbf{x}\left|e^{-t \tilde{K}}\right| \mathbf{x}\right\rangle$ up to the second order in $t$,

$$
\begin{aligned}
\left\langle\mathbf{x}\left|e^{-t \tilde{K}}\right| \mathbf{x}\right\rangle= & \left(\frac{1}{4 \pi t}\right)^{3 / 2}\left\{1+2 T^{3} B S_{z} t\right. \\
& \left.+\left(2\left(T^{3}\right)^{2} B^{2} S_{z}^{2}-\frac{\left(T^{3}\right)^{2} B^{2}}{6}\right) t^{2}+\cdots\right\},
\end{aligned}
$$

we deduce the following expression for $U(t)$ $\equiv\left\langle\mathbf{x}\left|e^{-t \widetilde{K}} \hat{Q}\right| \mathbf{x}\right\rangle$ :

[1] R. Jackiw, Analysis of Infinite-Dimensional ManifoldsSchrödinger Representation for Quantized Fields, Field Theory and Particle Physics (World Scientific, Singapore, 1990).

[2] L. I. Schiff, Phys. Rev. 130, 458 (1963); G. Rosen, ibid. 173, 1632 (1968).

[3] J. Kuti, J. Cornwall, R. Jackiw, and E. Tomboulis, Phys. Rev. D 10, 2428 (1974).

[4] T. Barnes and G. I. Ghandour, Phys. Rev. D 22, 924 (1980).

[5] W. A. Bardeen and M. Moshe, Phys. Rev. D 28, 1372 (1983).

[6] F. Cooper and E. Mottola, Phys. Rev. D 36, 3114 (1987).

[7] S. Y. Pi and M. Samiullah, Phys. Rev. D 36, 3128 (1987).

[8] O. Eboli, R. Jackiw, and S. Y. Pi, Phys. Rev. D 37, 3557 (1988)

[9] J. Greensite, Nucl. Phys. B158, 469 (1979).

[10] J. Cornwall, Phys. Rev. D 38, 656 (1988).

[11] M. Danos, D. Gogny, and D. Iracane, "Models for the NonPerturbative QCD vacuum,' U.S. Department of Commerce, National Institute of Standards and Technology, NISTIR No. 4555, 1991.

[12] A. K. Kerman and D. Vautherin, Ann. Phys. (N.Y.) 192, 408 (1989).

[13] R. P. Feynman, in Variational Calculations in Quantum Field Theory, Proceedings of the Workshop, Wangerooge, West Germany, 1987, edited by L. Polley and D. Pottinger (World Scientific, Singapore, 1988).

[14] I. I. Kogan and A. Kovner, Phys. Rev. D 52, 3719 (1995).

[15] W. E. Brown and I. I. Kogan, Int. J. Mod. Phys. A 14, 799 (1999).

[16] W. E. Brown, Int. J. Mod. Phys. A 13, 5219 (1998).

[17] K. Zarembo, Phys. Lett. B 421, 325 (1998); Mod. Phys. Lett. A 13, 2317 (1998).

$$
\begin{aligned}
U(t)= & \left(\frac{1}{4 \pi t}\right)^{3 / 2}\left\{\frac{2}{3}+T^{3} B S_{z} t\right. \\
& \left.+\left(\frac{7}{3}\left(T^{3}\right)^{2} B^{2} S_{z}^{2}-\frac{1}{3}\left(T^{3}\right)^{2} B^{2}\right) t^{2}+\cdots\right\} .
\end{aligned}
$$

Thus, finally,

$$
\begin{aligned}
\operatorname{Tr}\left\langle\mathbf{x}\left|G^{-1}\right| \mathbf{x}\right\rangle & =\frac{1}{\sqrt{\pi}} \int_{1 / \Lambda^{2}}^{\infty} \frac{d t}{t^{3 / 2}}[\operatorname{Tr}\langle\mathbf{x}|\hat{Q}| \mathbf{x}\rangle-\operatorname{Tr} U(t)] \\
& =(\cdots) \Lambda^{4}-\frac{C_{N}}{8 \pi^{2}}\left(\frac{11}{3} B^{2}\right) \ln \frac{\Lambda^{2}}{B}+\mathcal{O}\left(g^{2}\right)
\end{aligned}
$$

where $\langle\mathbf{x}|\hat{Q}| \mathbf{x}\rangle$ has been obtained by setting $t=0$ in Eq. (B20). This is the result announced in Eq. (5.18).
[18] D. Diakonov, lecture at the 4th St. Petersburg Winter School in Theoretical Physics, 1998, hep-th/9805137.

[19] D. J. Thouless and J. G. Valatin, Nucl. Phys. 31, 211 (1962).

[20] G. K. Savvidy, Phys. Lett. 71B, 133 (1977).

[21] F. M. H. Villars, in Proceedings of the International School of Physics “'Enrico Fermi,'” Varenna, 1966, edited by T. E. O. Ericson (Academic, New York, 1967).

[22] R. E. Peierls and J. Yoccoz, Proc. Phys. Soc. London A70, 381 (1957).

[23] P. Ring and P. Schuck, The Nuclear Many-Body Problem (Springer, Berlin, 1981).

[24] C. Heinemann, Ph.D. thesis, Université Pierre et Marie Curie, 2000.

[25] T. D. Lee, Particle Physics and Introduction to Field Theory (Harwood Academic, Chur, Switzerland, 1981), Sec. 18.5.

[26] K. Gottfried and V. F. Weisskopf, Concepts of Particle Physics (Oxford University Press, Oxford, 1986), Vol. II.

[27] N. K. Nielsen and P. Olesen, Nucl. Phys. B144, 376 (1978).

[28] H. B. Nielsen and P. Olesen, Nucl. Phys. B160, 380 (1979).

[29] A. Chodos, R. L. Jaffee, K. Johnson, C. B. Thorn, and V. F. Weisskopf, Phys. Rev. D 9, 3471 (1974).

[30] M. Shifman, A. Vainshtein, and V. I. Zakharov, Nucl. Phys. B147, 385 (1979).

[31] J. Schwinger, in Quantum Theory of Angular Momentum, edited by L. Biedenharn and H. Van Dam (Academic Press, New York, 1965).

[32] J. F. Dyson, Phys. Rev. 102, 1217 (1956).

[33] T. Holstein and H. Primakoff, Phys. Rev. 58, 1098 (1940).

[34] J. P. Blaizot and E. R. Marshalek, Nucl. Phys. A309, 422 (1978); Phys. Lett. 79B, 1 (1978).

[35] E. Brézin and C. Itzykson, Phys. Rev. D 2, 1191 (1970).

[36] J. Schwinger, Phys. Rev. 82, 664 (1951). 